\title{
Comparative Analysis on the Internal Force of CRD Method with different Steps for Excavating Super Shallow Tunnel
}

\author{
Weng Ling ${ }^{1, a}$, Zhang Dingbang ${ }^{1, b}$ \\ ${ }^{1}$ School of Civil Engineering, Hubei Polytechnic University, Huangshi, Hubei, 435003, China \\ a596009717@qq.com, b6076961@qq.com
}

Keywords: Super shallow tunnel, Axial force and moment, Tunnel excavation, CRD method.

\begin{abstract}
On the adaptability of CRD four steps method and six steps method for excavating super shallow tunnel, elastic-plastic simulation are executed by ANSYS and Mohr-coulomb criterion. The development and distribution of axial force and moment of two kinds of methods are compared. Analysis results indicate that the axial force and moment of CRD four steps method are larger than that of six steps method. It is comparatively obvious that the axial force and moment developed near the lateral contour of tunnel by both two methods. The CRD six steps method is better than four steps method for excavating super shallow tunnel and should be adopted in actual construction process.
\end{abstract}

\section{Introduction}

CRD excavating method is also called the medium diaphragm method, its construction technology is setting medium diaphragm in the middle of excavating section and divided each section into several parts, so as to reduce the excavation span and height. The characteristic of the method is high safety, less difficulty in construction, easily controlled ground settlement, and adapted to excavating tunnel in poor geological condition[1]. There are commonly two type of excavation steps using CRD method: four steps method and six steps method. Both the four steps method and six steps method have merit and demerit, the adaptability of CRD four steps method and six steps method had been studied by ANSYS in order to identify the most suitable excavation method for excavating super shallow tunnel.

\section{Engineering situation}

A urban metro is a one-portal double-track super shallow tunnel excavating by using CRD method and the buried depth is 4.5-9.2 m, excavation span is $15.3 \mathrm{~m}$, excavation height is $14.7 \mathrm{~m}$. The project is located in low mountains and hills district, and the direction of main structure line is northeast to southwest [2] [3]. The main engineering geological characteristics of the excavating area are as follows:

Table.1 Calculation Parameters

\begin{tabular}{|c|c|c|c|c|c|}
\hline $\begin{array}{c}\text { Surrounding rock } \\
\text { classification }\end{array}$ & $\begin{array}{c}\text { Elastic } \\
\text { modulus (Mpa) }\end{array}$ & $\begin{array}{c}\text { Poisson's } \\
\text { ratio }\end{array}$ & $\begin{array}{c}\text { Cohesion } \\
(\mathrm{kPa})\end{array}$ & $\begin{array}{c}\text { Internal friction } \\
\text { angle }\left({ }^{\circ}\right)\end{array}$ & $\begin{array}{c}\text { Density } \\
\left(\mathrm{kN} \cdot \mathrm{m}^{-3}\right)\end{array}$ \\
\hline Filled soils & 15.1 & 0.25 & 21.7 & 28.6 & 20.3 \\
\hline $\begin{array}{c}\text { Clay } \\
\text { Completely } \\
\text { decomposed granite }\end{array}$ & 17.3 & 0.34 & 35.1 & 25.1 & 25.7 \\
\hline Primary support & $2.3 \times 10^{-4}$ & 0.21 & - & 27.3 & 22.4 \\
\hline $\begin{array}{c}\text { Grouting } \\
\text { reinforcement }\end{array}$ & 73 & 0.22 & 50 & 22.4 & 22.0 \\
\hline
\end{tabular}

\section{Model Building}


Calculation Parameters. All kinds of soil and rock are assumed to obey the mohr-coulomb criterion, the main engineering calculation parameters of the excavating soil and rock are as Table 1.

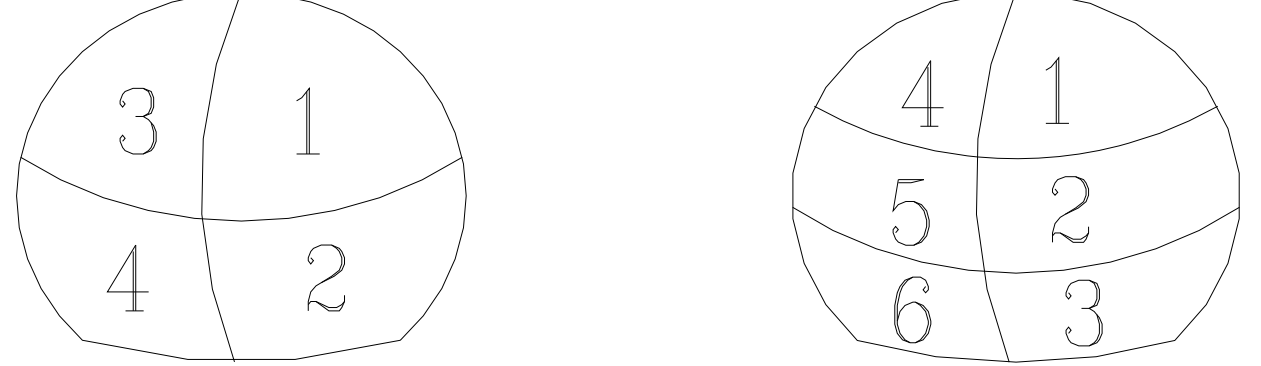

Fig.1 Excavation steps of four steps method

Fig.2 Excavation steps of six steps method

Model meshing. Calculating model and excavation steps are showed in Figure 1 and Figure 2.

In the course of modeling, two-dimensional 4-node isoparametric element is used to simulate the surrounding rock, primary support and other part of the tunnel[4].The finite element model of tunnel and surrounding rock is meshed with detailed units and grid size of the interior is smaller than boundary[5]. The CRD four steps excavation model is divided into 1450 finite elements and 1621 nodes, the six steps excavation model is divided into 1525 finite elements and 1734 nodes. The element grids of CRD four steps excavation model and six steps model are shown in figure 3 and figure 4.

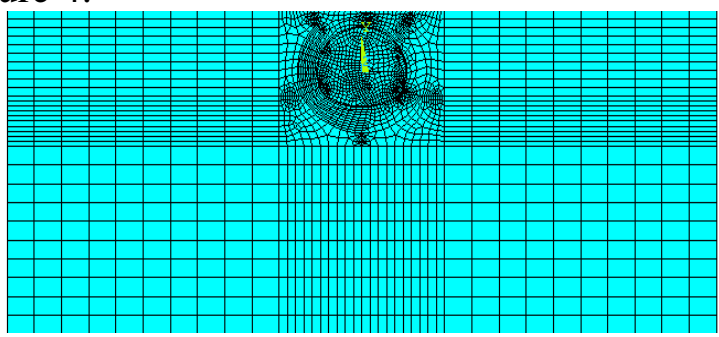

Fig.3 Grids of four steps excavation model

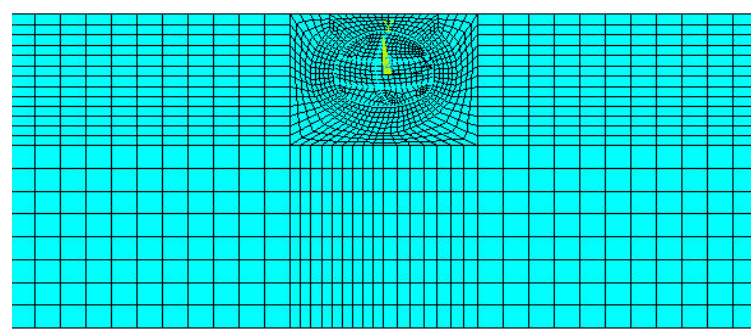

Fig.4 Grids of six steps excavation model

\section{Simulation data analysis}

In order to compare the adaptability of CRD four steps excavation method and six steps method, the development and distribution of axial force and moment of two kinds methods are analysised.

Axial force analysis. Axial force of four steps method and six steps method at the end of excavation are shown in figure 5 and figure 6, axial force of four steps method and six steps method after temporary support were demolished are shown in figure 7 and figure 8. From table 2, it's obvious that axial force of six steps method is smaller than that of four steps method both at the end of excavation and after temporary support were demolished. In the whole construction period adopting four steps method, the column of medium diaphragm bear small stress, the stress is mainly distribution at the bottom of medium diaphragm. In the whole construction period adopting six steps method, the column of medium diaphragm bear bigger stress than adopting six steps method. No matter which method is adopted, axial force at the temporary support will increase after the support were demolished and axial force at the right are bigger than the left.

Moment analysis. Moment of four steps method and six steps method at the end of excavation are shown in figure 9 and figure 10, moment of four steps method and six steps method after temporary support were demolished are shown in figure 11 and figure 12. From table 3, it's obvious that positive moment of six steps method is smaller than that of four steps method and negative moment of six steps method is bigger than that of four steps method both at the end of excavation and after temporary support were demolished. In the whole construction period adopting four steps method, the biggest positive moment appears at the left bottom of tunnel while the biggest negative moment appears at the bottom of medium diaphragm. The moment at the contour of tunnel is redistributed after temporary support were demolished, the biggest moment appears at the bottom and vault of the tunnel which will pay more attention in construction. 


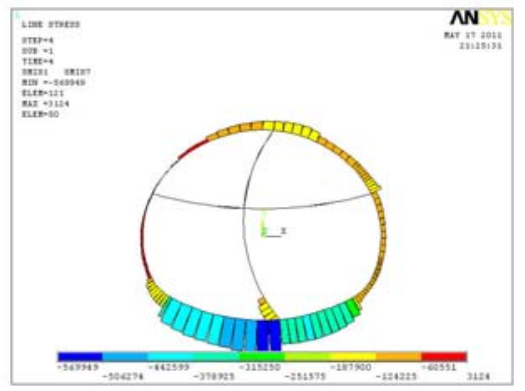

Fig. 5 Axial force of 4 steps method at the end of excavation

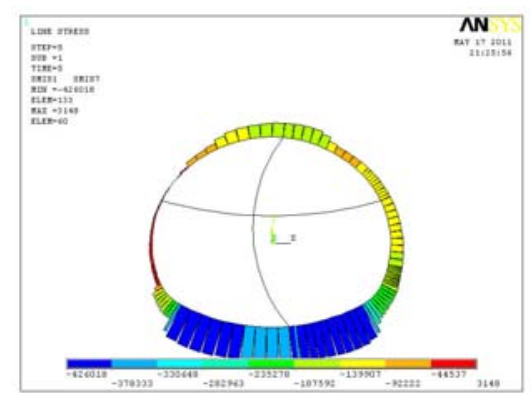

Fig.7 Axial force of 4 steps method without temporary support

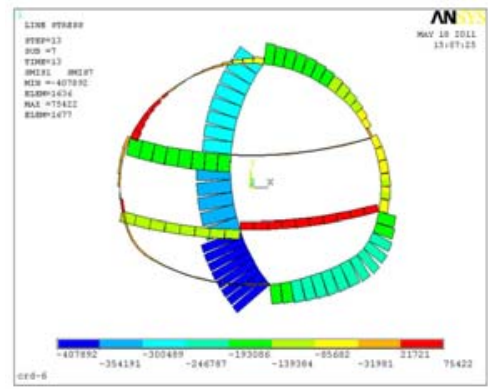

Fig.6 Axial force of 6 steps method at the end of excavation

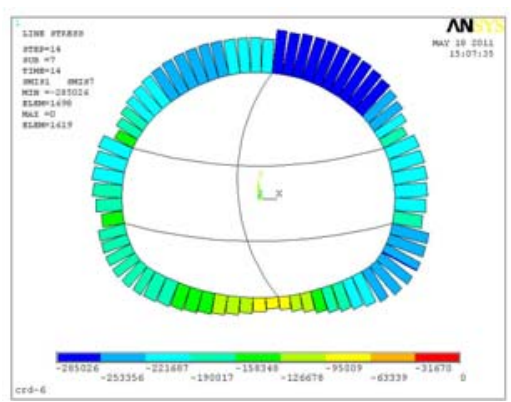

Fig.8 Axial force of 6 steps method without temporary support

Table.2 Axial force comparison

\begin{tabular}{|c|c|c|}
\hline Value & $\begin{array}{c}\text { Axial force after } \\
\text { excavation }(\mathrm{kN})\end{array}$ & $\begin{array}{c}\text { Axial force after support } \\
\text { were demolished(kN) }\end{array}$ \\
\hline Excavation method & 570 & 426 \\
\hline Six steps method & 407.9 & 285.0 \\
\hline
\end{tabular}

Table.3 Moment comparison

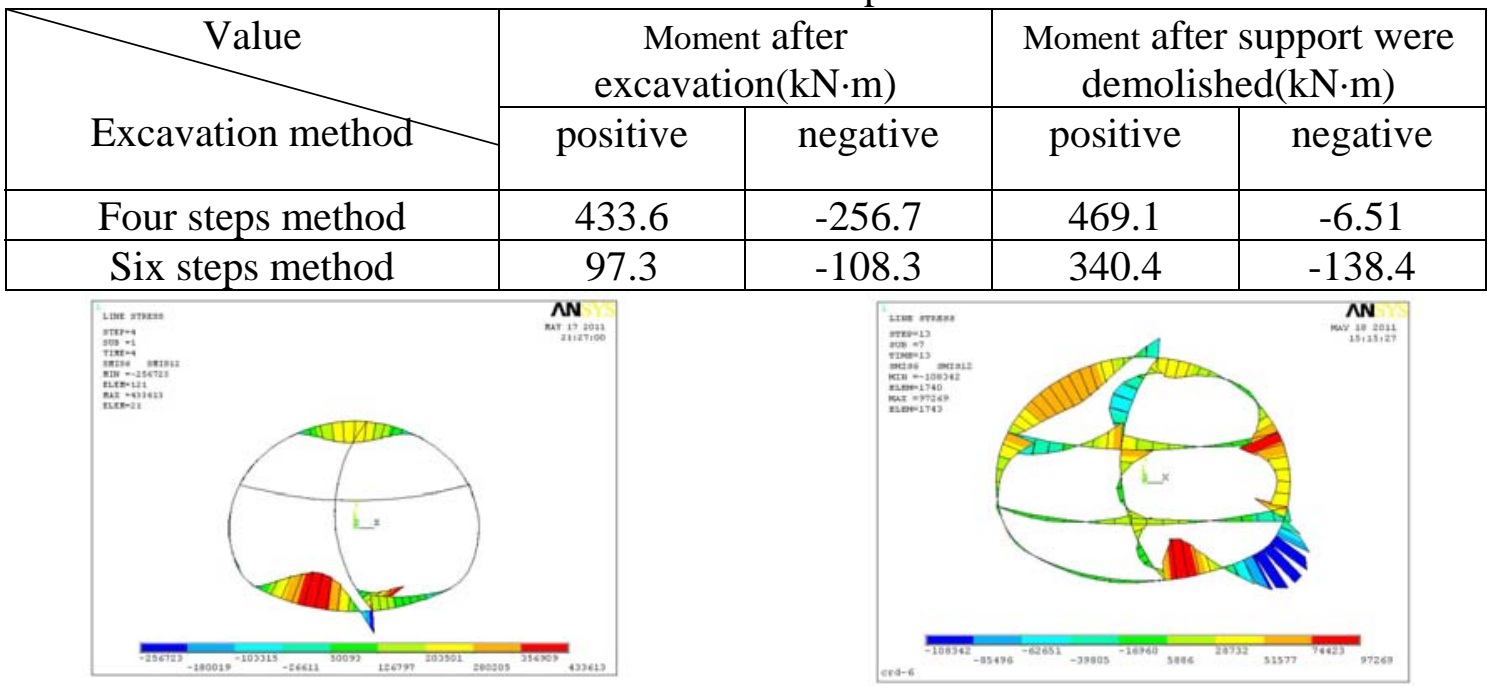

Fig.9 Moment of 4 steps method at the end of excavation
Fig.10 Moment of 6 steps method at the end of excavation

Based on the above analysis, it conclude that axial force and moment adopting six steps method is smaller than that of four steps method in the whole construction period, the reason is the excavation span and height of six steps method is smaller, using this method can reduce the disturbance of soil and control the deformation area. 


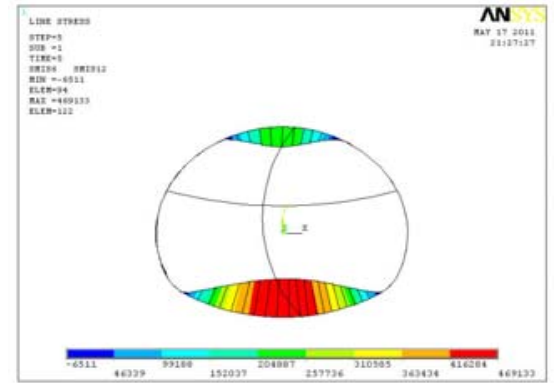

Fig.11 Moment of 4 steps method without temporary support

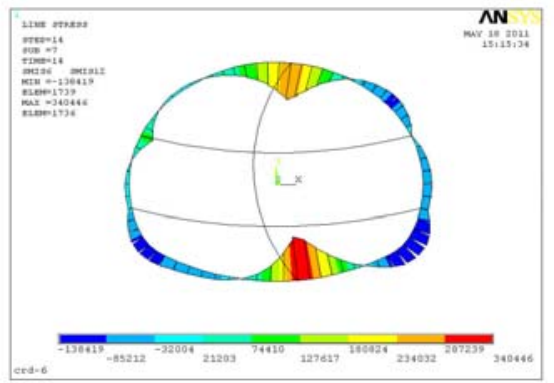

Fig.12 Moment of 6 steps method without temporary support

\section{Conclusions}

According to the comparative analysis on the internal force of CRD method with different steps for excavating super shallow tunnel, the following conclusion are made:

No matter adopting six steps method or four steps method, axial force at the temporary support will increase after the support were demolished and axial force at the right are bigger than the left.

The moment at the contour of tunnel is redistributed after temporary support are demolished, the biggest moment appears at the bottom and vault of the tunnel which will pay more attention in construction.

The axial force and moment adopting six steps method is smaller than that of four steps method in the whole construction period, so six steps method should be adopted in actual construction process for the tunnel safety.

\section{Acknowledgment}

The authors gratefully acknowledge the support of Teaching Research Project of Hubei Polytechnic University (Grant No. 201017) in this study.

\section{References}

[1] Huang, M.Q., FU X.L. and LI, Y.C. (2007). Study on Stability Control of Large-section Subsea Tunnels in Weak Strata by CRD Contruction Method. Chinese Journal of Rock Mechanics and Engineering, December, 3805-3809.

[2] Wang, W.M., Huang, M.Q. and Wu, K.X. (2007). The Numerical Analysis of Construction with CRD Method in Xiamen Xiangan Submarine Tunnel. Journal of Shandong University of Science and Technology(Natural Science), February, 27-31.

[3] Zhang, J.G., Wang, M.N. and Yu, S.Y. (2008). Analysis of Security of Temporary Supports by CRD Method in Xiamen East Passageway. Highway, March, 83-89.

[4] Liang, W., \& Huang, M.L. (2007). Subsidence and Deformation Control of Large-span Tunnel in Water-bearing Unfavorable Geological Conditions. Chinese Journal of Rock Mechanics and Engineering, December, 3738-3742.

[5] Guo, C., Yu S.Y. and Wang, M.N. (2009). The influence of excavation by sections with CRD method on the structural internal force and safety factor in undersea tunnel construction. Hydrogeology \& Engineering Geology, January, 75-79. 